\title{
Implementation of the Xpert MTB/RIF assay for tuberculosis in Mongolia: a qualitative exploration of barriers and enablers
}

\author{
Nicole L Rendell Corresp., ${ }^{1}$, Solongo Bekhbat ${ }^{2}$, Gantungalag Ganbaatar ${ }^{1}$ ， Munkhjargal Dorjravdan ${ }^{1}$, Madhukar \\ Pai $^{3}$, Claudia C Dobler ${ }^{4,5}$ \\ 1 National Tuberculosis Program Mongolia, Ulaanbaatar, Mongolia \\ 2 Mongolian Anti-Tuberculosis Association, Ulaanbaatar, Mongolia \\ 3 McGill International TB Centre, McGill University, Quebec, Canada \\ ${ }^{4}$ Woolcock Institute of Medical Research, University of Sydney, New South Wales, Australia \\ ${ }^{5}$ South Western Sydney Clinical School, University of New South Wales, New South Wales, Australia \\ Corresponding Author: Nicole L Rendell \\ Email address: nicole.rendell@gmail.com
}

Objective: The aim of our study was to identify barriers and enablers to implementation of the Xpert MTB/RIF test within Mongolia's National Tuberculosis Program.

Methods: Twenty-four semi-structured interviews were conducted between June and September 2015 with laboratory staff and tuberculosis physicians in Mongolia's capital Ulaanbaatar and regional towns where Xpert MTB/RIF testing had been implemented. Interviews were recorded, transcribed, translated and analysed thematically using NVIVO qualitative analysis software.

Results: Eight laboratory staff (five from the National Tuberculosis Reference Laboratory in Ulaanbaatar and three from provincial laboratories) and sixteen tuberculosis physicians (five from the Mongolian National Center for Communicable Diseases in Ulaanbaatar, four from district tuberculosis clinics in Ulaanbaatar and seven from provincial tuberculosis clinics) were interviewed. Major barriers to Xpert MTB/RIF implementation identified were: lack of awareness of program guidelines; inadequate staffing arrangements; problems with cartridge supply management; lack of local repair options for the Xpert machines; lack of regular formal training; paper based system; delayed treatment initiation due to consensus meeting and poor sample quality. Enablers to Xpert MTB/RIF implementation included availability of guidelines in the local language; provision of extra laboratory staff, shift working arrangements and additional modules; capacity for troubleshooting internally; access to experts; opportunities for peer learning; common understanding of diagnostic algorithms and decentralised testing.

Conclusion: Our study identified a number of barriers and enablers to implementation of Xpert MTB/RIF in the Mongolian National Tuberculosis Program. Lessons learned from this study can help to facilitate implementation of Xpert MTB/RIF in other Mongolian locations as well as other low-and middle-income countries. 


\section{Implementation of the Xpert MTB/RIF assay for tuberculosis}

\section{2 in Mongolia: a qualitative exploration of barriers and enablers}

3

4 Nicole Rendell $^{1}$, Solongo Bekhbat ${ }^{2}$, Gantungalag Ganbaatar ${ }^{1}$, Munkhjargal Dorjravdan ${ }^{1}$,

5 Madhukar Pai ${ }^{3}$, Claudia C Dobler 4,5

6

Corresponding Author: Nicole Rendell - nicole.rendell@gmail.com

${ }^{1}$ National Tuberculosis Program Mongolia, Ulaanbaatar, Mongolia

${ }^{2}$ Mongolian Anti-Tuberculosis Association, Ulaanbaatar, Mongolia

${ }^{3}$ McGill International TB Centre, McGill University, Quebec, Canada

${ }^{4}$ Woolcock Institute of Medical Research, University of Sydney, New South Wales, Australia

${ }^{5}$ South Western Sydney Clinical School, University of New South Wales, New South Wales, Australia 


\section{Abstract}

Objective: The aim of our study was to identify barriers and enablers to implementation of the Xpert MTB/RIF test within Mongolia's National Tuberculosis Program.

Methods: Twenty-four semi-structured interviews were conducted between June and September 2015 with laboratory staff and tuberculosis physicians in Mongolia's capital Ulaanbaatar and regional towns where Xpert MTB/RIF testing had been implemented. Interviews were recorded, transcribed, translated and analysed thematically using NVIVO qualitative analysis software.

Results: Eight laboratory staff (five from the National Tuberculosis Reference Laboratory in Ulaanbaatar and three from provincial laboratories) and sixteen tuberculosis physicians (five from the Mongolian National Center for Communicable Diseases in Ulaanbaatar, four from district tuberculosis clinics in Ulaanbaatar and seven from provincial tuberculosis clinics) were interviewed. Major barriers to Xpert MTB/RIF implementation identified were: lack of awareness of program guidelines; inadequate staffing arrangements; problems with cartridge supply management; lack of local repair options for the Xpert machines; lack of regular formal training; paper based system; delayed treatment initiation due to consensus meeting and poor sample quality. Enablers to Xpert MTB/RIF implementation included availability of guidelines in the local language; provision of extra laboratory staff, shift working arrangements and additional modules; capacity for troubleshooting internally; access to experts; opportunities for peer learning; common understanding of diagnostic algorithms and decentralised testing.

Conclusion: Our study identified a number of barriers and enablers to implementation of Xpert MTB/RIF in the Mongolian National Tuberculosis Program. Lessons learned from this study can 
41 help to facilitate implementation of Xpert MTB/RIF in other Mongolian locations as well as

42 other low-and middle-income countries. 


\section{Introduction}

44 In December 2010, the World Health Organization (WHO) formally endorsed use of Xpert

45

MTB/RIF as a new diagnostic tool for active tuberculosis (TB) disease. ${ }^{1}$ Since then, use of Xpert MTB/RIF has increased dramatically. Between 2010 and 2015, under concessional pricing arrangements, 4,672 GeneXpert machines had been procured in 122 of the 145 eligible countries, including Mongolia. ${ }^{2}$

Mongolia, a country with a TB burden of 428 incident cases per 100,000 population in 2015 (95\% confidence interval $220-703)^{3}$, has been a relatively late adopter of the Xpert MTB/RIF assay. In Mongolia, the rate of concomitant HIV and TB is low at 0.34 incident cases per 100,000 population (95\% Cl: $0.26-0.44)$, but the country has a relatively high burden of multi-drug resistant (MDR) and rifampicin resistant (RR) TB cases representing $2.2 \%(95 \% \mathrm{Cl}: 1.1-3.3)$ of new cases and 33\% (95\% Cl: 29-38) of previously treated cases. ${ }^{3}$ Resistance against all first-line drugs tested is approximately $60 \%$ among sputum smear-positive patients in whom standard first-line TB treatment failed, suggesting successful transmission (versus new mutation) of these highly resistant strains in the community. ${ }^{4}$ At the time of the first introduction of MTB/RIF in Mongolia at the end of 2013 , other countries had already analysed their experiences with implementation of Xpert MTB/RIF. ${ }^{5}$ It was, however, not until 2014 that the WHO published a 'How-To' Xpert MTB/RIF implementation manual. ${ }^{6}$ It is therefore of interest to explore the specific challenges with Xpert MTB/RIF implementation in Mongolia. 
64 The National Tuberculosis Reference Laboratory (NTRL) in Ulaanbaatar was the first laboratory

65 in Mongolia to implement Xpert MTB/RIF testing in November 2013. By the end of 2014, there

66 were three GeneXpert machines being used in Mongolia - at the NTRL in Ulaanbaatar, the

67 Hospital of Darkhan-uul, in the northern Darkhan-uul province, near the Russian border, (since

68 June 2014) and the Regional Diagnostic and Treatment Center of Dornod, in Dornod province,

69 Mongolia's easternmost province, bordering Russia and China (since March 2014). The funding

70 for these machines was supplied by the Global Fund through a series of projects. In 2013, 310

71 patient samples were tested using Xpert MTB/RIF. This had increased to 3,289 in 2014, 3,802 in

722015 and 3,991 in 2016 (Table 1).

Diagnostic tools such as Xpert MTB/RIF must be considered in the context of their

organisational environment to understand the value of their technical benefits in real terms.

Such benefits include the relatively short time frame to determine a result ( 2 hours) and ease of use compared to other diagnostic methods. Understanding the operational issues associated with implementation of the Xpert MTB/RIF test ensures these technical benefits can be maximised.

In recent years a number of studies have assessed the performance of the Xpert MTB/RIF assay, specifically, the accuracy and cost effectiveness of Xpert MTB/RIF have been well documented. ${ }^{7-12}$ Only few research studies, however, have investigated implementation issues from an organisational perspective, ${ }^{5,13-17}$ two of which included qualitative tools designed specifically for the study to gather information on user experiences and challenges related to 
Xpert MTB/RIF implementation. ${ }^{5,16}$ Some of the identified challenges associated with Xpert MTB/RIF implementation in low-and middle income countries include lack of standardised guidance, piecemeal implementation of training, quality assurance, planning processes and equipment servicing and maintenance, issues with continuous power supply and difficulties recording and reporting test results using new technology. $5,16,17$

The aim of our study was to identify and understand system and context specific factors within Mongolia's National Tuberculosis Program (NTP) that are barriers or enablers to implementing the Xpert MTB/RIF test from the perspective of NTP staff.

Methods

\section{Study design, setting and participants}

We conducted semi-structured interviews with laboratory staff and TB physicians using an inductive-deductive approach. This approach was chosen because we had knowledge about possible barriers and enablers to GeneXpert implementation from studies in other settings, $5,13-$

${ }^{15}$ while we knew little about contextual factors impacting on Xpert MTB/RIF implementation in Mongolia. We developed semi-structured interview guides (see Appendix A) to collect a wide range of information on participants' experiences and views related to Xpert MTB/RIF use and implementation in Mongolia's NTP. The interview guide was designed based on a literature review of barriers and enablers of Xpert MTB/RIF implementation. These implementation factors were organised into major themes that formed the structure of the interview guide for 
107 laboratory staff - guidelines and organisational structures; equipment; training; communication

108 systems; and diagnostic algorithms, case finding for Xpert MTB/RIF, clinical management. A

109 separate interview guide was designed specifically for TB physicians and focused on clinical

110 issues. Open questions were added to the interview guide to explore possible themes that had

111 not been identified from the literature. Two of the researchers (GG and MD) conducted the

112 interviews. Both interviewers were trained in the study protocol and interview technique.

113 Participants included laboratory staff (doctors, technicians and administrative officers) who

114 were using the Xpert MTB/RIF diagnostic test and TB physicians who were working either at the

115 Mongolian National Center for Communicable Diseases (NCCD) or in any of the district TB clinics

116 in Mongolia's capital Ulaanbaatar, or in the provinces of Darkhan-Uul or Dornod.

Potential participants were approached by one of the researchers (GG or MD) and interviewed

once informed consent was obtained. Because there were only few laboratory staff who were

using the Xpert MTB/RIF diagnostic test, all of them were invited to participate in the study.

Interviews with TB physicians were continued until saturation of data was apparent, that is until

no new themes emerged. ${ }^{18}$ All interviews were conducted between July and September 2015.

124 Participants in Ulaanbaatar were interviewed in person where possible, or alternatively over

125 the phone, and participants based outside of Ulaanbaatar were interviewed over the phone.

126 The interviews were conducted and transcribed in Mongolian. The transcripts were then

127 translated into English by one of the researchers (SB). The English transcripts were verified by 
128 another bi-lingual member of the research team (MD) to ensure the English version was clear

129 and the participants' views were adequately represented.

\section{Analysis}

132 In order to determine whether participants would interpret interview questions as intended and whether the order of questions may influence responses, we conducted three pretesting interviews (with two laboratory staff and one TB physician). Pretesting highlighted questions that were poorly understood by respondents. It also showed that some questions were perceived by respondents to be duplicate questions. We edited the interview guides accordingly by reordering, rewording or deleting questions. In addition, pretesting showed that explanatory information provided by the interviewer varied considerably between interviews. In response to this observation, a written introduction explaining the purpose and benefit of the study was added to the interview instrument for the interviewers to read aloud before the interview commenced. This ensured consistent explanatory information was given to all participants prior to the commencement of the interview. The revised interview guides were used for all future interviews. The pretest interviews were not included in the final analysis.

The first (NR) and last author (CCD) independently reviewed transcripts from the first five interviews to identify key themes and subthemes (ie, patterns within the narrative data), then developed a coding scheme through discussion and consensus. This framework was systematically applied to code themes in subsequent interview transcripts. We reviewed the

149 framework after an additional ten interviews, updating it to reflect newly revealed themes and 
150 subthemes that were not apparent in earlier interviews. Coding was completed using the

151 software package QSR NVivo version 10 (QSR International Pty Ltd, Doncaster, Victoria,

152 Australia). The English version of each interview transcript was imported into NVivo and then

153 systematically reviewed and coded for common themes and subthemes.

154 The study results are reported in accordance with the Consolidated Criteria for Reporting

155 Qualitative Research (COREQ) checklist. ${ }^{19}$

157 Ethics

158 The study was approved by the Scientific Council of the Mongolian NCCD.

\section{Results}

161 We contacted 24 NTP staff (8 laboratory staff and 16 TB physicians), all of whom agreed to be

162 interviewed (ie, a $100 \%$ response rate). Five laboratory staff members were located at the NTRL

163 in Ulaanbaatar and 3 in provincial laboratories. Of the 16 TB physicians, 5 worked at the NCCD,

1644 worked in any of the district TB clinics in Ulaanbaatar and 7 worked in the provinces of

165 Darkhan-Uul or Dornod.

166

167 Participants identified a range of barriers and enablers associated with implementation of Xpert

$168 \mathrm{MTB} /$ RIF testing in Mongolia based on their experiences. These are summarised in Table 2, and

169 described below according to the following themes: 1) guidelines and organisational structures, 
170 2) equipment, 3) training, 4) communication systems, 5) diagnostic algorithms, case finding for

171 Xpert MTB/RIF and clinical management. Laboratory staff participants were interviewed using

172 guiding questions for each theme. For TB physicians, the guiding questions focussed on the last

173 theme, diagnostic algorithms, case finding for Xpert MTB/RIF and clinical management.

175 Guidelines and organisational structures Barriers

Poor awareness of program guidelines: Around half of all participants stated that they were not aware of any written guidelines to support the use of Xpert MTB/RIF (authors' comment: the Mongolian NTP issued guidelines for all NTP staff, which included information on the use of Xpert MTB/RIF in December 2014). Instead, many participants referred to training arrangements when asked about NTP guidelines. One participant thought that the English manual for the operation of the Xpert machine was equivalent to the NTP guidelines available to staff. The guidelines did not appear to be clear enough about which samples (type of body fluid/tissue) could be used for testing with Xpert MTB/RIF, in particular if testing of samples other than sputum, e.g. pleural and cerebrospinal fluid, was allowed. This resulted in inconsistent practices regarding Xpert MTB/RIF testing of non-sputum samples (authors'comment: According to the Mongolian NTP guidelines 2014 the following specimens can be used for Xpert MTB/RIF testing: sputum, urine, stool, pleural fluid, ascites, gastric lavage, and surgical tissue samples, although the protocol for collecting and transporting specimens collected for testing was not included in the guidelines at the time of the study). One laboratory 
192 (inconsistent with guideline recommendations, summarised in Table 3), which was potentially

193 driving excess demand.

194 Inadequate staffing arrangements: Participants identified that their workload had increased in

195 response to the introduction of the Xpert MTB/RIF testing into their laboratories, and

196 inadequate staffing was mentioned as a problem by staff of the NTRL in Ulaanbaatar.

$197 \quad$ Enablers

198 Clear guidelines in local language: Participants, who were aware of the NTP guidelines, found

199 the guidelines useful for working with Xpert MTB/RIF and they understood how to apply the

200 guidelines in practice.

201 Extra staff, shift working arrangements, increase of Xpert MTB/RIF modules: Staff of the

202 regional laboratories felt that while there was an initial increase in their workload, their staffing

203 arrangements and laboratories were able to accommodate the change. Staff of the NTRL in

204 Ulaanbaatar suggested a range of options to improve their ability to meet the demand. These

205 included additional staff, a staff member allocated only to Xpert MTB/RIF, shift arrangements,

206 installing more GeneXpert machines or a new GeneXpert machine with a greater number of

207 modules (in order to perform more tests at the same time).

208

\section{Equipment}

Barriers

Poor supply chain management of cartridges (stock-outs): Since implementation of Xpert

MTB/RIF testing, the only time the test had been unavailable for about a month was due to a cartridge supply issue that affected all the laboratories that offered Xpert MTB/RIF testing. 
214 Other than this occasion, cartridge supply appeared to be well managed (sufficient number of

215 cartridges available to meet demand without cartridge expiration issues due to oversupply).

216 Absence of local repair options: Mongolia's harsh climate and low population density generally

217 did not appear to have affected transportation of cartridge supplies or equipment. Although,

218 one of the modules in one of the regional machines had broken on arrival and there were

219 difficulties arranging its repair. The lack of expertise to repair GeneXpert machines in the

220 provinces was identified as a problem. This was in contrast to other laboratory equipment,

221 which could be repaired by the available engineers.

Enabler

Capacity for troubleshooting internally: Other temporary problems with the GeneXpert

machine have occurred without major interruption to the workflow because local staff were able to remedy them easily. For example, one laboratory participant mentioned that the laboratory had bought an uninterruptable power supply to manage issues with the power supply. Another laboratory participant mentioned the need for more basic office equipment to cater for the introduction of the new equipment.

\section{Training}

\section{Barrier}

Inconsistent formal training options: The training that laboratory staff had received on the operation of Xpert MTB/RIF varied widely. Some laboratory staff participated in formal training courses, others learned on the job through instruction by trained colleagues or superiors. Two members of laboratory staff based in Ulaanbaatar participated in an online training course 
236 organised by the supplier of the GeneXpert machines. Components of different trainings

237 included operation and maintenance of GeneXpert, but also information on indications for

238 GeneXpert use in Mongolia.

239 When asked about the value of the training received, most laboratory staff felt that it was

240 sufficient to meet their job requirements. While staff in Ulaanbaatar were satisfied that the

241 training they had received equipped them to use Xpert MTB/RIF testing, regional laboratory

242 staff were keen to receive further training.

243 There were also differences in the training opportunities provided for other TB diagnostic

244 methods, primarily smear testing. Laboratory staff were aware of scheduled annual trainings

245 for smear testing, but were unsure about any future and/or regular ongoing training

246 opportunities for Xpert MTB/RIF testing.

$247 \quad$ Enablers

248 Access to experts: Access to external technical experts to support implementation of Xpert

249 MTB/RIF differed for staff based in regional clinics and those based in Ulaanbaatar. Regional

250 laboratory staff received guidance from NTRL staff, who received guidance directly from

251 international experts on operations and troubleshooting of the machines.

252 Peer learning: All staff recognised the value of learning from their peers.

254 Communication systems

Barrier

Paper based system: Results of the Xpert MTB/RIF test were communicated to TB clinics using paper forms that were delivered using one or more of the following options: 1 ) patients 
258 collected the paper form directly from the laboratory and took it to the doctor at the TB clinic,

259 2) doctors were contacted over the phone and informed about the results ahead of receiving

260 the paper form, and 3) doctors and/or nurses collected the results on paper directly from the

261 laboratory. Paper reports were associated with delays of 2 days on average to receive the

262 results following a request being sent to the laboratory. A few TB physicians suggested the

263 delay was longer, up to a week, and one TB physician even suggested that the delay between

264 requesting Xpert MTB/RIF testing to receiving the result was up to 2 weeks. Urgent requests

265 were prioritised.

266 Administrative reports were generated weekly on a computer, based on the information on the

267 paper forms that was manually input into a computer file. Despite having regular administrative

268 reporting of the results to facilitate a quality control mechanism, participants did not value the

269 reporting system in this way. It is unclear based on the interview data how the information

270 contained in the reports could be used to prompt action to improve processes.

271

272 Diagnostic algorithms, case finding for Xpert MTB/RIF and clinical management

$273 \quad$ Barriers

274 Treatment initiation in MDR-TB delayed until after consensus meeting: Treatment for drug

275 sensitive cases (who tested positive for TB on GeneXpert, but negative for rifampicin resistance)

276 was usually started immediately after physicians received the results. TB physicians described

277 that the delay to initiating treatment for presumptive MDR-TB (following a positive result for

278 rifampicin resistance), as well as the specifics of the MDR-TB treatment regimen depended on

279 the timing of the weekly MDR-TB meeting (held at the central NTP office in Ulaanbaatar, to 
280 discuss each case of MDR-TB in Mongolia and decide on the best approach for the patient's

281 treatment). Some participants reported that there was no delay in commencing treatment

282 following a positive test for rifampicin resistance. Others reported that it took 1 to 2 weeks to

283 initiate treatment because the treatment could not commence until the treatment plan had

284 been agreed upon at the MDR-TB meeting.

285 Poor sample quality: 'Error results' from Xpert MTB/RIF testing (i.e., notification of an error is

286 displayed in the Check Status screen of the GeneXpert machine) were reported to occur

287 occasionally. In these instances, the test was always redone and some participants reported

288 that error results triggered machine maintenance as well. The quality of the sample (e.g. when

289 patients did not collect sputum correctly) was reported as the most common reason for error

290 results experienced by participants when using Xpert MTB/RIF testing. Some laboratory staff

291 participants also reported seeking additional training and/or assistance to minimise error

292 results.

293

Enablers

294

Common understanding of indications for Xpert MTB/RIF testing: Both laboratory staff and TB

physicians were aware of the approved indications for Xpert MTB/RIF use in Mongolia (Table 3),

including testing in patients with possible MDR-TB (patients with TB relapse, positive sputum smear result at the $3^{\text {rd }}$ and $5^{\text {th }}$ month of treatment and screening of MDR-TB close contacts), as

ill patients with uncertain diagnosis). 
300 There was variation in the details of each participant's response regarding indications for

301 GeneXpert use, but broadly the indications as outlined above were consistent among

302 participants and consistent with Mongolian guidelines (see table 3).

303 Testing availability in provincial centres (decentralised): Participants from the provinces

304 pointed out that while Xpert MTB/RIF testing is available in regional areas of Mongolia, samples

305 need to be sent to the central reference laboratory in Ulaanbaatar for further drug

306 susceptibility testing from culture. They did, however, still value access to Xpert MTB/RIF

307 testing, because it meant that a rapid diagnosis of drug resistant TB was possible in the regional

308 areas when previously, it could only be done in Ulaanbaatar.

309 Most physicians had not experienced difficulties starting their patients on MDR-TB treatment

310 following a diagnosis of rifampicin resistance. However, those that did reported that the reason

311 they could not commence treatment was because of patient related factors rather than

312 program related issues.

\section{Suggestions for future Xpert MTB/RIF implementation and scale up}

315 All study participants viewed the new diagnostic technology positively and appreciated the time saving benefits unique to the Xpert MTB/RIF test. When asked about what future changes they would like to see to implementation of Xpert MTB/RIF testing in Mongolia, participants highlighted the need for change in the following three areas:

- Increase in the number of available sites for Xpert MTB/RIF testing (upscaling to more districts of Ulaanbaatar and more provinces in Mongolia) 
321

322

323

324

325

326

327

328

329

330

331

332

333

334

335

336

337

- Use of Xpert MTB/RIF to test for drug resistance on all smear positive patients before starting treatment

- Increase in the number of machines at existing sites.

\section{Discussion}

This study highlighted a range of potential factors within the Mongolian NTP that served as a

barrier or enabler to the implementation of Xpert MTB/RIF testing. Since the implementation of Xpert MTB/RIF testing in Mongolia, according to NTP data, the number of notified cases detected in Ulaanbaatar, Darkhan and Dornod has increased from 2,783 cases in 2012 to 3,029 cases in 2015. While all study participants appreciated the benefits of the new diagnostic technology and were supportive of its implementation, all participants were able to identify areas where integration into the existing program could be improved. Potential barriers included lack of awareness of program guidelines; inadequate staffing arrangements; problems with cartridge supply management; lack of local repair options for the Xpert machines; lack of regular formal training; paper based system; delayed treatment initiation due to consensus meeting and poor sample quality. Enablers included availability of guidelines in the local language; provision of extra laboratory staff, shift working arrangements and additional modules; capacity for troubleshooting internally; access to experts; opportunities for peer learning; common understanding of diagnostic algorithms and decentralised testing. Lessons learned from this study can inform the implementation and upscaling of GeneXpert in other settings in Mongolia and in other low-and middle income countries. 
343 Policies and guidelines as well as training opportunities provided by an organisation facilitate

344 the uptake of any new technology. ${ }^{6}$ In our study, those that knew about the NTP's guidelines for

345 the use of GeneXpert found them to be invaluable. However, only around half of study

346 participants were aware of any guidelines relating to GeneXpert use and relied on random

347 opportunities for training and/or visits from national and international experts to advise them

348 on the use of Xpert MTB/RIF. Adherence to program guidelines, which should incorporate Xpert

349 MTB/RIF testing into the national diagnostic strategy and algorithms, ${ }^{6}$ is important for

350 sustainable implementation of Xpert MTB/RIF in a local context. Diagnostic algorithms and

351 other policies outlined in program guidelines are essential to realise and maximise the potential

352 of new technologies in practice. ${ }^{20-22}$

353

354 Only staff from NTRL, but not from the regional laboratories, had concerns about their capacity

355 to meet the demand for Xpert MTB/RIF testing. This is most likely because the regular requests

356 for the population that the NTRL laboratory covered went above their pre-existing surge

357 capacity. In contrast, the regional laboratories only felt under pressure initially, while they were

358 still learning how to use the technology. A feasibility study conducted in India also found that in

359 a decentralised setting, there were minimal infrastructure modifications and human resource

360 concerns following the implementation of Xpert MTB/RIF testing. ${ }^{15}$ The lower population

361 density in settings covered by existing laboratories in the decentralised and regional areas,

362 potentially allowed the introduction of new diagnostic technology using the pre-existing surge

363 capacity of the laboratory workforce.

364 
365 Issues with the infrastructure supporting Xpert MTB/RIF testing were observed by many

366 laboratory staff and focused on the one month where cartridge supply was an issue, and to a

367 lesser extent, access to repairs. In our study the demand for cartridges was met without

368 expiration issues due to high turnover of cartridges, except for one month when the cartridge

369 supply was exhausted. No Xpert MTB/RIF testing could be undertaken during this month.

370 Previous studies investigating Xpert MTB/RIF implementation have highlighted the importance

371 of program levers to assist in the management of cartridge supply. ${ }^{5,14}$ They have suggested

372 measures such as staggered cartridge shipments and design of diagnostic algorithms that

373 combine Xpert with other methods to increase yield. ${ }^{5,14} \mathrm{~A}$ Brazilian study assessing the pilot

374 implementation of Xpert MTB/RIF calculated that $10 \%$ of Xpert MTB/RIF equipment needed

375 replacement during the eight month pilot study, yet spare parts were not immediately

376 available. ${ }^{14}$ Although our study did not quantify faulty equipment, a similar experience of

377 limited access to repairs was observed by laboratory staff. The authors of the Brazilian study

378 suggested that both cartridge supply and maintenance should be negotiated with

379 manufacturers. ${ }^{14}$

380

381

Communication of Xpert MTB/RIF results occurred through different methods that were paper

based and reliant on the accuracy of the contact information written on the Xpert MTB/RIF

request form. Interestingly, no participant would have preferred computer based record

keeping or an online system, even though administrative reports were generated weekly on a

computer, based on the information in the paper forms, manually input into the computer files. 
387 mechanism that would be of value to the participants. A study undertaken in two Brazilian

388 cities found that the implementation of concomitant IT technology to support Xpert MTB/RIF

389 integration into organisational workflows, led to delays in physicians receiving laboratory

390 results. ${ }^{16}$ In that study, difficulties with the online system were thought to be a result of poor

391 knowledge on how to use the equipment and networks effectively, low availability of online

392 systems and available systems not interacting with each other leading to repeated input of

393 data. ${ }^{16}$ Given the limited availability and uptake of electronic databases and online systems

394 within Mongolia's NTP, it is reasonable to assume that Mongolian NTP staff may have concerns

395 about upscaling IT technology that echo the experience outlined in the Brazilian study. ${ }^{16}$

396 However, IT solutions enable more efficient record keeping internally and capacity for

397 connectivity externally to pool surveillance data that can benefit TB control efforts within the 398 region. ${ }^{23}$

399

400 Several studies have highlighted issues with sample quantity and quality for GeneXpert testing. ${ }^{5,14,15}$ Our findings are consistent with these studies - participants suggested the most common reason for experiencing an error message was related to the sample's quantity and/or quality. Specimen transportation systems are in place at the three levels of the health system community, district/provincial and central. All health care workers with responsibility for collecting specimens receive training on the collection and effective transportation of

406 specimens. When collecting specimens, patients are provided with verbal and written 407 instructions. Participants also reported conflicting information on the type of specimen that 
408

409

410

411

412

413

414

415

416

417

418

419

420

421

422

423

424

425

426

427

428

429

could be used for Xpert MTB/RIF testing, indicating the need for better education and training in this area.

The rapid testing feature of Xpert MTB/RIF (results available in 2 hours) means that the technology is poised for point of care (POC) testing. Our study supports the findings of studies on POC testing that found that simply having the rapid test technology available does not guarantee a natural fit into the program environment to enable POC testing. ${ }^{13,24-26}$ Detailed recommendations on how to facilitate integration of GeneXpert with clinical care have been made. ${ }^{27}$ Two of these recommendations, standardised training and establishment of automated processes for prescriptions, ${ }^{27}$ would address important barriers identified in our study.

In our study, processes that followed a diagnosis of MDR-TB contributed to time delays until treatment initiation despite the rapid availability of Xpert MTB/RIF results. An Indian study on a POC testing program found that diagnostic delays, such as those observed in our study, undermined the full potential of rapid tests. ${ }^{28}$ However, two trials suggest that the benefit of prompt treatment initiation gained using Xpert MTB/RIF may not translate into improved patient outcomes. One randomised controlled trial found that nurses in African primary care clinics had the capacity to accurately administer Xpert MTB/RIF at the clinic, which resulted in more patients starting same-day treatment, more culture-positive patients starting therapy and a shorter time to treatment initiation compared to the use of sputum smear microscopy. ${ }^{29}$ However, the trial also found that these benefits did not translate into lower tuberculosis related morbidity. ${ }^{29}$ The other trial, a South African cluster-randomised trial, found that there 
was no reduction in mortality at six months when using Xpert MTB/RIF compared to smear

431 microscopy. ${ }^{30}$ In Mongolia, Xpert MTB/RIF is currently not implemented as a POC test, as the

432 laboratories performing the testing are not directly integrated with the TB clinics. Improving

433 integration of GeneXpert with clinical care by addressing some of the issues outlined above

434 could potentially increase the value of GeneXpert testing.

More broadly, funding arrangements and health system functioning have also been identified in

the literature to have an important role in the implementation of new diagnostic

technologies. ${ }^{31}$ However, these concepts were outside the scope of this study because we

439 focused on the experiences of operational staff.

A limitation of our study was that interview questions and answers had to be translated from

English into Mongolian and from Mongolian into English respectively, possibly resulting in loss

or misinterpretation of information. To reduce these risks, we pretested the interview guides and adjusted them based on participant feedback, and the English interview transcripts were verified by a second bi-lingual member of the research team to ensure the English version was

clear and the participants' views were adequately represented. The interviews were semistructured with open-ended questions, which allowed respondents to explore the mentioned topics as they saw fit. Adding more structured interview questions to explore some issues in more detail (e.g. sample collection, distance and subsequent travel time of samples, transport conditions particularly during winter and waste disposal system for cartridges) might have been useful. 
452 In summary, this study identified a number of barriers and enablers of Xpert MTB/RIF

453 implementation in Mongolia that extended beyond purchasing the equipment and installing it

454 locally. The program environment is important for the successful implementation of Xpert

455 MTB/RIF. Our study found that factors affecting implementation centred around awareness of

456 program guidelines, cartridge supply management, local repair options for GeneXpert

457 technology, regular formal training, communication systems and processes for sample

458 collection. Upscaling of Xpert MTB/RIF testing facilities in Mongolia and other low-and middle

459 income countries will lead to implementation of Xpert MTB/RIF in new settings in future, and

460 we believe that the lessons learned from our study can help to facilitate this process.

461

462 Acknowledgements

463 This work was supported (in-kind) by the NTP of Mongolia. We thank Dr Buyankhishig

464 Burneebaatar for her assistance during the ethics approval process and sourcing background

465 information. 


\section{References}

467

468 1. World Health Organization. WHO endorses new rapid tuberculosis test [Internet]. Geneva (Switzerland): World Health Organization; media release 8 December 2010 [cited 17 November 2016]. Available from http://www.who.int/mediacentre/news/releases/2010/tb test 20101208/en/

2. World Health Organization. Global Tuberculosis Report 2016 [Internet]. Geneva (Switzerland): World Health Organization; 2016 [cited 17 November 2016]. Available from http://apps.who.int/iris/bitstream/10665/250441/1/9789241565394eng.pdf?ua=1

3. World Health Organization. Mongolia - Tuberculosis profile [Internet]. Geneva $\underline{\text { ROD/EXT/TBCountryProfile\&ISO2=mn\&outtype }=\text { pdf }}$

4. Dobler CC, Korver S, Batbayar O, Nyamdulam B, Oyuntsetseg S, Tsolmon B, Surmaajav B, Bayarjargal B, Marais BJ. Multidrug-Resistant Tuberculosis in Patients for Whom First-Line Treatment Failed, Mongolia, 2010-2011. Emerg Infect Dis. 2015; 21(8): 1451-1454 [cited 21 May 2017]. Available from https://www.ncbi.nlm.nih.gov/pubmed/26196504 
programmatic implementation of Xpert MTB/RIF testing in nine countries. BMC

488

Infect Dis. 2014; 14(2): p. 2. doi 10.1186/1471-2334-14-2

489

6. World Health Organization. Xpert MTB/RIF Implementation Manual: Technical and

Operational 'How-To'; Practical Considerations [Internet]. Geneva (Switzerland):

491

World Health Organization; 2014 [cited 5 December 2016]. Available from:

492

https://www.ncbi.nlm.nih.gov/books/NBK254329/

7. World Health Organization. Xpert MTB/RIF assay for the diagnosis of pulmonary and

extrapulmonary TB in adults and children - Policy Update [Internet]. Geneva

495

(Switzerland): World Health Organization; 2013 [cited 17 November 2016]. Available

496

from http://apps.who.int/iris/bitstream/10665/112472/1/9789241506335 eng.pdf

497

8. Armand S, Vanhuls P, Delcroix G, Courcol R, Lemaître N. Comparison of the Xpert

498

MTB/RIF test with an IS6110-TaqMan real-time PCR assay for direct detection of

499

Mycobacterium tuberculosis in respiratory and nonrespiratory specimens. J Clin

500

Microbiol. 2011; 49(5): 1772-6. doi 10.1128/JCM.02157-10

501

9. Nhu NT, Ha DTM, Anh ND, Thu DD, Duong TN, Quang ND, Lan NT, Quyet TV, Tuyen

502

NT, Ha VT, Giang DC, Dung NH, Wolbers M, Farrar J, Caws M. Evaluation of Xpert

503

504

MTB/RIF and MODS assay for the diagnosis of pediatric tuberculosis. BMC Infect Dis.

2013; 13: 31. doi 10.1186/1471-2334-13-31

10. Huh HJ, Jeong B, Jeon K, Koh WJ, Ki CS, Lee NY. Performance evaluation of the Xpert

506 MTB/RIF assay according to its clinical application. BMC Infect Dis. 2014; 14: 589. doi 
508

509

510

511

512

513

514

515

516

517

518

519

520

521

522

523

524

525

526

527

528

11. Menzies NA, Cohen T, Lin H, Murray M, Salomon JA. Population health impact and cost-effectiveness of tuberculosis diagnosis with Xpert MTB/RIF: a dynamic simulation and economic evaluation. PLoS Med. 2012; 9(11): e1001347. doi 10.1371/journal.pmed.1001347

12. Vassall A, van Kampen S, Sohn H, Michael JS, John KR, den Boon S, Davis JL, Whitelaw A, Nicol MP, Gler MT, Khaliqov A, Zamudio C, Perkins MD, Boehme CC, Cobelens F. Rapid diagnosis of tuberculosis with the Xpert MTB/RIF assay in high burden countries: a cost-effectiveness analysis. PLoS Med. 2011; 8(11): e1001120. doi:10.1371/journal.pmed.1001120

13. Engel N, Davids M, Blankvoort N, Pai NP, Dheda K, Pai M. Compounding diagnostic delays: a qualitative study of point-of-care testing in South Africa. Trop Med Int Health. 2015; 20(4): 493-500. doi 10.1111/tmi.12450

14. Durovni B, Saraceni V, Cordeiro-Santos M, Cavalcante S, Soares E, Lourenço C, Menezes A, van den Hof S, Cobelens F, Trajman A. Operational lessons drawn from pilot implementation of Xpert MTB/Rif in Brazil. Bull World Health Organ. 2014; 92(8): 613-7. doi http://dx.doi.org/10.2471/BLT.13.131409

15. Raizada N, Sachdeva KS, Sreenivas A, Vadera B, Gupta RS, Parmar M, Kulsange S, Babre A, Thakur R, Gray C, Ramachandran R, Alavadi U, Ghedia M, Vollepore B, Dewan P, Boehme C, Paramsivan CN. Feasibility of decentralised deployment of Xpert MTB/RIF test at lower level of health system in India. PLoS One. 2014; 9(2): e89301. doi 10.1371/journal.pone.0089301 
529 16. de Camargo Jr KR, Guedes CR, Caetano R, Menezes A, Trajman A. The adoption of a new diagnostic technology for tuberculosis in two Brazilian cities from the perspective of patients and healthcare workers: a qualitative study. BMC Health Serv Res. 2015; 15: 275. doi 10.1186/s12913-015-0941-x

17. Albert H, Nathavitharana RR, Isaacs C, Pai M, Denkinger CM, Boehme CC. Development, roll-out and impact of Xpert MTB/RIF for tuberculosis: what lessons have we learnt and how can we do better? Eur Respir J. 2016; 48: 516-525. doi $10.1183 / 13993003.00543-2016$

18. Patton MQ. Qualitative evaluation and research methods. Newbury Park (CA): Sage Publications Inc; 1990.

19. Tong A, Sainsbury P, Craig J. Consolidated criteria for reporting qualitative research (COREQ): a 32-item checklist for interviews and focus groups. Int J Qual Health Care. 2007; 19(6):349-57 [cited 21 May 2017]. Available from https://www.ncbi.nlm.nih.gov/pubmed/17872937

20. McNerney R, Cunningham J, Hepple P, Zumla A. New tuberculosis diagnostics and rollout. Int J Infect Dis. 2015; 32: 81-86. doi: 10.1016/j.ijid.2015.01.012

21. Kirwan DE, Cárdenas MK, Gilman RH. Implementation of New TB Diagnostic Tests: Is It Too Soon for a Global Roll-Out of Xpert MTB/RIF? Am J Trop Med Hyg. 2012; 87(2): 197-201. doi:I0.4269/ajlmh.2012.12-0I07 
evaluation of GeneXpert for the diagnosis of HIV- negative pediatric TB cases. BMC Infect Dis. 2015; 15:70. doi 10.1186/s12879-015-0814-2

552 23. Andre E, Isaacs C, Affolabi D, Alagna R, Brockmann D, de Jong BC, Cambau E, Churchyard G, Cohen T, Delmee M, Delvenne JC, Farhat M, Habib A, Holme P, Keshavjee S, Khan A, Lightfoot P, Moore D, Moreno Y, Mundade Y, Pai M, Patel S, Nyaruhirira AU, Rocha LE, Takle J, Trébucq A, Creswell J, Boehme C. Connectivity of

24. Cowan J, Michel C, Manhiça I, Monivo C, Saize D, Creswell J, Gloyd S, Micek M. Implementing rapid testing for tuberculosis in Mozambique. Bull World Health Organ. 2015; 93: 125-130. doi 10.2471/BLT.14.138560

25. Pai NP, Vadnais C, Denkinger C, Engel N, Pai M. Point-of-Care Testing for Infectious Diseases: Diversity, Complexity, and Barriers in Low- And Middle-Income Countries. PLoS Med. 2012; 9(9): e1001306. Doi 10.1371/journal.pmed.1001306

26. Clouse K, Page-Shipp L, Dansey H, Moatlhodi B, Scott L, Bassett J, Stevens W, Sanne I, Van Rie A. Implementation of Xpert MTB/RIF for routine point-of-care diagnosis of tuberculosis at the primary care level. S Afr Med J. 2012; 102(10): 805. doi 10.7196/SAMJ.5851

27. Dominique JK, Ortiz-Osorno AA, Fitzgibbon J, Gnanashanmugam D, Gilpin C, Tucker T, Peel S, Peter T, Kim P, Smith S. Implementation of HIV and Tuberculosis Diagnostics: The Importance of Context. Clin Infect Dis. 2015; 61(S3): S119-25. doi $10.1093 /$ cid/civ552 
572 28. Engel N, Ganesh G, Patil M, Yellappa V, Vadnais C, Pai NP, Pai M. Point-of-care testing in India: missed opportunities to realize the true potential of point-of-care testing programs. BMC Health Serv Res. 2015; 15: 550. doi 10.1186/s12913-0151223-3

29. Theron G, Zijenah L, Chanda D, Clowes P, Rachow A, Lesosky M, Bara W, Mungofa S, Pai M, Hoelscher M, Dowdy D, Pym A, Mwaba P, Mason P, Peter J, Dheda K; TBNEAT team. Feasibility, accuracy, and clinical effect of point-of-care Xpert MTB/RIF testing for tuberculosis in primary-care settings in Africa: a multicentre, randomised, controlled trial. Lancet. 2014; 383: 424-35. doi 10.1016/S0140-6736(13)62073-5

30. Churchyard GJ, Stevens WS, Mametja LD, McCarthy KM, Chihota V, Nicol MP, Erasmus LK, Ndjeka NO, Mvusi L, Vassall A, Sinanovic E, Cox HS, Dye C, Grant AD, Fielding KL. Xpert MTB/RIF versus sputum microscopy as the initial diagnostic test for tuberculosis: a cluster-randomised trial embedded in South African roll-out of Xpert MTB/RIF. Lancet Glob Health. 2015; 3: e450-57. doi 10.1016/S2214-

31. ngel N, Wachter K, Pai M, Gallarda J, Boehme C, Celentano I, Weintraub R.

doi:10.1136/bmjgh-2016-000132

32. Ministry of Health Mongolia. 1st Annex of the Ministerial order of the Ministry of Health 592 (N319) - Manual on the Management of Tuberculosis care and services. 2014; Ulaanbaatar: Ministry of Health Mongolia. 
595 Supporting Information

596 S1 File. Appendix A - Interview Guides 
597 Table 1. Number of patient specimens tested using Xpert MTB/RIF, by laboratory, in

598 Mongolia, 2013-2014

\begin{tabular}{|c|c|c|c|c|c|}
\hline \multirow{2}{*}{$\begin{array}{l}\text { Laboratory } \\
\text { location }\end{array}$} & \multirow[b]{2}{*}{ Commencement } & \multicolumn{4}{|c|}{ Number of patient specimens ${ }^{1}$ tested } \\
\hline & & 2013 & 2014 & 2015 & 2016 \\
\hline Ulaanbaatar & November 2013 & 310 & 3,022 & 3,493 & 3,588 \\
\hline Dornod & March 2014 & - & 130 & 114 & 162 \\
\hline Darkhan-uul & June 2014 & - & 137 & 195 & 241 \\
\hline
\end{tabular}

599

$600{ }^{1}$ Specimens include all pulmonary and extra-pulmonary specimens 
Table 2. Barriers and enablers of Xpert MTB/RIF implementation, 2015

\begin{tabular}{|l|l|}
\hline Barriers & Enablers \\
\hline
\end{tabular}

\section{Guidelines and organisational structures}

- Poor awareness of program guidelines - staff members were not

- Clear guidelines in local language - in situations where participants always aware that guidelines existed and how they could be accessed

- Inadequate staffing arrangements - laboratory staff participants indicated an increase in their workload without a change in staffing arrangements to accommodate the increased workload. were aware of guidelines in Mongolian, they were considered valuable guidance for working with Xpert MTB/RIF

- Extra staff in NTRL, shift working arrangements and/or an increase in the number of modules - these arrangements would have assisted staff in meeting the increased demands that resulted from the introduction of Xpert MTB/RIF.

\section{Equipment}

- Poor supply chain management of cartridges (stock-outs) - this

- Capacity for troubleshooting internally - there where some situations happened on one occasion and meant Xpert MTB/RIF testing where the participants were able to determine the cause of machine ceased.

- Absence of local repair options - difficulties were reported for faults and resolve them using a locally sourced solution. This meant little interruption to the work flow. arranging repairs when required because of limited availability of trained mechanics to repair Genexpert machines in Mongolia. 


\begin{tabular}{|c|c|}
\hline Barriers & Enablers \\
\hline \multicolumn{2}{|c|}{ Training } \\
\hline $\begin{array}{l}\text { - Inconsistent formal training options - some laboratory staff } \\
\text { participated in formal training courses, others learned on the job } \\
\text { through instruction by trained colleagues or superiors. }\end{array}$ & $\begin{array}{l}\text { - Access to experts - some participants had direct access to visiting } \\
\text { international technical experts } \\
\text { - Peer learning - all staff recognised the value of learning from their } \\
\text { colleagues. }\end{array}$ \\
\hline \multicolumn{2}{|c|}{ Communication Systems } \\
\hline $\begin{array}{l}\text { - Paper based system - storing patient information on paper forms } \\
\text { through the laboratory workflow meant results were } \\
\text { communicated inefficiently through a paper based system and } \\
\text { administrative reporting required a manual input of information. }\end{array}$ & \\
\hline \multicolumn{2}{|c|}{ Diagnostic algorithms, case finding for Xpert MTB/RIF and clinical management } \\
\hline $\begin{array}{l}\text { - Treatment initiation in MDR-TB delayed until after consensus } \\
\text { meeting - some participants reported a delay in initiating MDR-TB } \\
\text { treatment because of the procedural requirement to determine } \\
\text { the MDR-TB treatment plan at a weekly meeting in Ulaanbaatar. } \\
\text { - Poor sample quality - this was the most commonly reported error } \\
\text { experienced by study participants. }\end{array}$ & $\begin{array}{l}\text { - Common understanding of indications for Xpert MTB/RIF testing } \\
\text { (diagnostic algorithms) - all participants reported an awareness of the } \\
\text { indications for Xpert MTB/RIF use in Mongolia } \\
\text { - Testing availability in provincial centres (decentralised) - participants in } \\
\text { provincial clinics value the accessibility of a tool to diagnose MDR-TB } \\
\text { locally. }\end{array}$ \\
\hline
\end{tabular}




\begin{tabular}{|c|c|}
\hline Indication & Additional detail \\
\hline \multicolumn{2}{|l|}{ All smear negative pulmonary TB cases } \\
\hline \multicolumn{2}{|l|}{$\begin{array}{l}\text { Patient with presumed pulmonary TB } \\
\text { diagnosed with HIV/AIDS }\end{array}$} \\
\hline Patients with presumed MDR-TB & $\begin{array}{l}\text { - Smear positive at the } 2^{\text {nd }}\left(3^{\text {rd }}\right) \text { and } 5^{\text {th }} \text { month of TB } \\
\text { treatment with category I and II } \\
\text { - Smear positive after interruption of TB treatment } \\
\text { category I and II } \\
\text { - } \text { Relapse (after TB treatment category I and II) } \\
\text { - Indefinite previous treatment regimen or smear } \\
\text { negative } \\
\text { - Smear positive after being smear negative during } \\
\text { treatment initiation } \\
\text { - Identification of TB case from contact } \\
\text { investigation of a DR-TB case. }\end{array}$ \\
\hline Patients with presumed XDR-TB & $\begin{array}{l}\text { - Used category II drugs for } 2 \text { or more months } \\
\text { - Culture positive at the } 3^{\text {rd }} \text { month of MDR-TB } \\
\text { treatment } \\
\text { - } \\
\text { treat converted to negative at the end of intensive } \\
\text { - Smear positive again by bacteriological analysis } \\
\text { after conversion to negative during continuous } \\
\text { treatment phase of MDR-TB } \\
\text { - MDR-TB case defined to be resistance to } \\
\text { fluoroquinolone group or second line injectable } \\
\text { TB drugs } \\
\text { Close contacts of XDR-TB case. }\end{array}$ \\
\hline $\begin{array}{l}\text { All smear positive new cases aged } 15- \\
34 \text { years old (this guideline is yet to be } \\
\text { implemented) }\end{array}$ & \\
\hline
\end{tabular}

